\title{
Positionen der FMH zur sogenannten Bedarfsplanung gemäss Art. 55a KVG
}

\section{Ausgangslage}

1. Die sogenannte Bedarfsplanung nach Art. 55a KVG wird auf dem Verordnungsweg eingeführt; sie kann aktuell weder politisch noch juristisch in Frage gestellt werden. Dies gilt insbesondere für die politische Ebene, wo jede Intervention an der seltenen Einhelligkeit von EDI und kantonalen Gesundheitsdirektoren scheitern würde.

2. Die Verordnung ist durch eine Vielzahl ungeklärter methodischer und operativer Fragen belastet. Diese können angesichts der Tatsache, dass die Verordnung nach gemeinsamem Willen des EDI und der kantonalen Gesundheitsdirektoren baldmöglichst eingeführt werden soll, nicht mehr bereinigt werden.

3. Der zentrale Einwand ist und bleibt die Tatsache, dass die Verordnung zu einer nachhaltigen, schweren Benachteiligung der jungen Ärztinnen und Ärzte mit zusätzlich drohender Überalterung der Ärzteschaft führt. Dieser Einwand ist umso gewichtiger, als nie nachgewiesen wurde, dass die Zulassungsbeschränkung für die Praxis ihr Ziel, nämlich eine Verflachung des Kostenwachstums, wirklich erreicht.

\section{Forderungen}

Die FMH sichert trotz dieser schweren Bedenken ihre Mitarbeit bei der Umsetzung dieser Vorlage unter folgenden Bedingungen zu:

a. Die Wirkungsdauer der Verordnung wird bis spätestens 31. Dezember 2003 bzw. 18 Monate nach Inkrafttreten angesetzt.

b. Diese Zeit wird ausgenützt, um eine flexible, den medizinischen Interessen unserer Bevölkerung entsprechende und alle Leistungserbringer gleichermassen betreffende Bedarfsplanung auszuarbeiten.

c. Der Bund leitet sofort Massnahmen ein, die der Erarbeitung einer Positivliste dienen; diese gibt Auskunft, welche Leistungen durch die obligatorische Grundversicherung zu bezahlen sind (Leistungspflicht).

d. Der Gesetzgeber verzichtet aktuell auf eine Modifikation des Vertragszwanges und fördert die Ausschöpfung der gegebenen gesetzlichen Möglichkeiten.

Die Verbindung Schweizer Ärztinnen und Ärzte FMH ist sich bewusst, dass die mögliche zusätzliche Eröffnung von 300 Arztpraxen durch in der Schweiz schon ansässige ausländische Ärztinnen und Ärzte zu einem schweren, medizinisch nicht gerechtfertigen Kostenanstieg führen müsste; in diesem Sinne akzeptiert sie die Einführung der Verordnung als Notmassnahme zu den oben ausgeführten Konditionen. Sie ist sich bewusst, dass sie ihren jungen Mitgliedern damit einen schweren Tribut abfordert, sichert diesen aber zu, sie bei Kampfmassnahmen vollumfänglich $\mathrm{zu}$ unterstützen, sollten die oben zusammengefassten Bedingungen nicht erfüllt werden. 